\title{
Analyzing the interactions between fluid and solid particles
}

\author{
Sherko Ahmad Flamarz Al-Arkawazi
}

Sulaimani Polytechnic University (SPU), Al- Sulaimaniyah, Kurdistan Region, Iraq Email: sherko.flamarz@spu.edu.iq

\begin{abstract}
This study focuses on analyzing and modeling the interactions between fluid and solid particles. A model based on the detection of contacts in granular medium is developed from a Discrete Elements approach (DEM) for solid phase, coupled with a Computational Fluid Dynamics (CFD) for fluid phase. The objective of this work is to investigate the interactions occurs between the fluid and solid particles in fluidized beds, and to better understand the various characteristic of these interactions which is the base of work of unit operations in a set of industrial processes. A comparison between different models of fluid flow (laminar model, $k-\varepsilon$ model, and $k-\omega$ SST model) in CFD code is showed that; the $k-\varepsilon$ model is most appropriate for calculation of fluid flow in industrial applications. The interaction between the fluid and each particle is performed through a drag force. The effect of the local particle concentration on the drag force is modeled by a porosity function. The simulations results are revealed that the value of exponent of porosity shows substantial dependence on the size of the Representative Volume Element (REV), tortuosity and the velocity of the fluid flow. Finally, the comparison between numerical simulation results with experimental results, in terms of fluidized bed height are showed that the bed expansion height of the fluidized bed is increased with an increase of fluid velocity, and this presents already a very good fit, which eventually achieve an optimization of fluidization processes.
\end{abstract}

Keywords: Interaction fluid-solid; Drag force; Fluidization; Coupling DEM-CFD

\section{Introduction}

The multiphase flow is essential in the work of many of industrial operations and processes, in which a close contact between the fluid and the solid particles. Therefore, the fluid-solid interaction plays a major role in unit processes, including, fluidization, catalytic cracking, sedimentation, particle classification, pharmaceutical, food processing, water treatments, chemical reactions, pneumatic conveying, crystallization, and so on (Matheson et al., 1949; Richardson \& Zaki, 1954; Hartge \& Werther, 1986; Bürger \& Wendland, 2001; Hirano et al., 2013). The fluidization processes or fluidized 
beds are a typical example of fluid-solid particle interaction; therefore, the fluidized beds are suited for chemical reactions as they allow exchanging the hydrodynamic and mechanic energies between the fluid and the solid particles. These conditions make it possible obtaining better yields and also greater selectivity. Besides, the use of fluidized beds makes it possible to obtain a high homogeneity of distribution of the solid phase and a high efficiency of the exchanges of energies.

Since 1920, the fluidization processes are used in the industrial applications, so a large number of researches are having been provided to improve and develop the fluidization units. The majority of the researches were experimental investigations of the characteristics and transport phenomena in the fluidization processes. The practical experiments include; devices and probes of measurements that disturb the fluidized beds and there behaviors and herewith they effect the result of the measurement. The practical limitations had overcome through computer models, since the development of the computer science in early of 1990s. The computers models are able to study and simulate from inside (microscopic interactions between particle-particle and fluidparticle) without disturb the fluidized beds.

The numerical study of fluidized beds through coupling the discrete code (discrete element method DEM) with continuum code (computational fluid dynamics CFD), enables to simulate (measure) many of properties, such as particle velocity, porosity, height of the fluidized beds and the interactions forces, as well as the velocity of the fluid, which are very difficult, if not impossible, to obtain by direct experimental probes. Many of researchers are proposed models to combine the DEM model with the CFD codes, such as Tsuji et al. (1993) and Hoomans et al. (1996), in which they are reported the coupling of the DEM with a finite volume description of the gas-phase based on the Navier-Stokes equations for the soft-sphere model and the hard sphere model respectively. Yu \& Xu (2003), are studied the vital role of the interaction forces between the particles in expanded bed. Zhu et al., (2008) are achieved to work in the particle scale simulation by combining DEM approach with CFD approach, respectively for solid and fluid phases. The coupling models of DEM-CFD have proved an ability to achieve and reproduce most of the features of the fluidized beds for complex units that includes multiphase flows (Chavan et al., 2018; Di Maio \& Di Renzo, 2007; Gidaspow, 1994; Apostolou \& Hrymak, 2008; Wang et al., 2010; Chu et al., 2018; Al-Arkawazia et al., 2017).

Although the advantages of the numerical models, but they are still need to more analysis in large scale industrial units of fluid-solid particles, to well understanding of the fundamentals of fluid-particle flows (Deen et al., 2007). Especially the behaviors 
which are related to the collision forces that results from the inter-particle reactions and the drag force produced from fluid-particle interaction.

In this work, the coupling of the two approaches (DEM-CFD) is used for analyzing and simulating the hydrodynamics behaviors of fluid-solid particles, through the fluidization process. The motion of the solid particles are treated by the discrete element method (DEM) is based on the Newtonian equations of motion, and the collision forces between the particle-particle (and wall) are modeled by hard particle approach (Fortain \& De Saxcé, 1999). The fluid flow is calculated by using the computational fluid dynamics (CFD), which is based on the Navier-Stokes equations (Archambeau et al., 2004). This is why we analyze and study numerically the interaction between fluid-solid particles through the drag force during the fluidization process, in order to propose a model of estimation of the best size of REV and value of the exponent of porosity correction. In a second step, we will integrate this model with a complete calculation of fluidization, which will be confronted with experimental results, in terms of height of bed expansion according to the inlet velocity of the fluid.

The work presented herein is arranged as follows; we gives and introduction of general principles relating to the discrete element method (DEM), the computational fluid dynamics (CFD), the coupling of DEM-CFD, and the governing equations in section 2 . We validate the DEM-CFD model by simulating a single particle, for different models of flow in CFD (laminar, $k-\varepsilon$, and $k-w$ SST) for a range of fluidization velocities, and compare the value of drag force calculated by these models of CFD with the value of drag calculated by theoretical equation. Then we simulate and evaluate the hydrodynamics behaviors of the fluid-solid particles interactions through a comparison between the numerical results with the experimental results to find the best size of REV and the exponent of porosity value $(n)$ in section 3 . Finally, we discuss and analyze the results and conclude the points that are carried out in the presented work.

\section{Material and Methods}

A good prediction of the characteristics of a fluid-solid mixture requires the knowledge to understand, and analyze the governing equations of fluid phase and solid phase. The behavior of fluidized beds is controlled by the effective forces acting on the motion of the solid particles; these forces are mainly the inter-particle contact forces, gravitational force, buoyancy force, and drag force, as shown in Fig.1. In present work, the DEM code is developed in our laboratory, and the CFD code is the open source software code Saturne (version 4), which is developed by electricity of France (EDF) (Archambeau et al., 2004). 


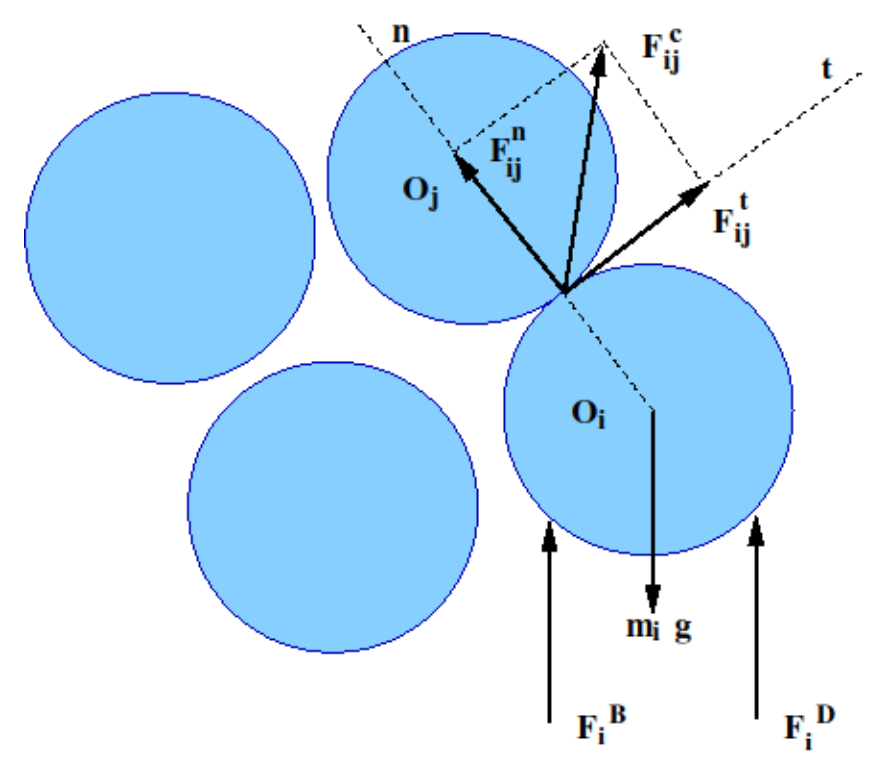

Figure 1: Schematic of the forces acting on particle $i$ from contacting particle $j$ and fluid-particle interactions.

\subsection{Governing equations of particle motion}

In the DEM code, the motion of each individual particle is governed by Newton's second law (linear momentum conservation and angular momentum), expressed, for the $i$-particle, by:

$$
\begin{aligned}
& m_{i} \frac{d \vec{v}_{i}}{d t}=\sum_{j}^{n c}\left(\vec{F}_{i j}^{n}+\vec{F}_{i j}^{t}\right)+m_{i} \vec{g}+\vec{F}_{i}^{B}+\vec{F}_{i}^{D} \\
& I_{i} \frac{d \vec{\omega}_{i}}{d t}=\sum_{j}^{n c}\left(\vec{r}_{i} \times \vec{F}_{i j}^{t}\right)
\end{aligned}
$$

where $m_{i}, \vec{v}_{i}$ represent, mass, velocity of the ( $i$ th) particle respectively, $n c$ is the number of contacts, $\vec{F}_{i j}^{n}$ and $\vec{F}_{i j}^{t}$ are the normal and tangential contact forces exerted by the neighboring particles on particle $i$ which is calculated by the DEM code, $\vec{g}$ is the gravitational acceleration, $\vec{F}_{i}^{B}$ is the buoyancy force, $\vec{F}_{i}^{D}$ is the drag force exerted by the fluid on the particle, $I_{i}, \vec{\omega}_{i}$, and $\vec{r}_{i}$ are the moment of inertia, angular velocity, and radius of a particle respectively.

\subsection{Governing equations of fluid flow}


The CFD code used in this work is the open source code Saturne, which solves the Reynolds-Averaged Navier-Stokes (RANS) equations (Archambeau et al., 2004). The continuity and momentum equations are governing the fluid flow, and can be written as:

$$
\begin{aligned}
& \frac{\partial \rho_{f}}{\partial t}+\vec{\nabla} \cdot\left(\rho_{f} \vec{v}_{f}\right)=0 \\
& \frac{\partial\left(\rho_{f} \vec{v}_{f}\right)}{\partial t}+\vec{\nabla} \cdot\left(\rho_{f} \vec{v}_{f} \otimes \vec{v}_{f}\right)=\vec{\nabla} \cdot(\vec{\sigma})+S_{u}
\end{aligned}
$$

where $\rho_{f}, \vec{v}_{f}$ are the density and velocity vector for the flow fluid respectively, $\vec{\sigma}$ is the stress tensor. For a turbulent flow, the stress tensor $\sigma$ includes the effect of pressure, viscous stress $\tau$, the turbulent Reynolds stress tensor $R_{i j}$, and $S_{u}$ represents additional momentum source terms. In the present work, it was chosen to use; laminar model, $k-\varepsilon$ model and $k-\omega$ SST (Shear Stress Transport) model (Menter, 1994). The laminar model is used the Navier-Stokes equations, Among the turbulence closure models available in the code, it was made the choice of using the first order two-equation models, $k-\varepsilon$ model and $k-\omega$, largely applied for their simplicity. The transport of the turbulent kinetic energy $k$ and dissipation rate $\varepsilon$ is examples of equations commonly used as closure, leading to the well-known standard $k-\varepsilon$ turbulence model (Mohammadi \& Pironneau, 1994).

$$
\begin{aligned}
& \rho_{f} \frac{\partial k}{\partial t}+\nabla \cdot\left(\rho_{f} \vec{v}_{f} k-\left(\mu_{f}+\frac{\mu_{t}}{\sigma_{k}}\right) \operatorname{grad} k\right)=P+G-\rho_{f} \varepsilon \\
& \frac{\partial \varepsilon}{\partial t}+\nabla \cdot\left(\rho_{f} \vec{v}_{f} \varepsilon-\left(\mu_{f}+\frac{\mu_{t}}{\sigma_{\varepsilon}}\right) \operatorname{grad} \varepsilon\right)=C_{\varepsilon 1} \frac{\varepsilon}{k}\left[P+\left(1-C_{\varepsilon 3}\right) G\right] P-\rho_{f} C_{\varepsilon 2} \frac{\varepsilon^{2}}{k} \\
& v_{t}=\frac{\mu_{t}}{\rho_{f}}=C_{\mu} \frac{k^{2}}{\varepsilon}
\end{aligned}
$$

$k$ is the turbulent kinetic energy, $\varepsilon$ the turbulent dissipation, $\mu_{f}$ the fluid dynamic viscosity, $\mu_{t}$ the turbulent viscosity, $P$ accounts for the production of the kinetic energy through mean shear stresses, $G$ the production term related to gravity effects and finally $\sigma_{k}=1, \sigma_{\varepsilon}=1.3, C_{\varepsilon l}=1.44, C_{\varepsilon 2}=1.92$ and $C_{\mu}=0.09$ defined constants. $C_{\varepsilon 3}=0$ if $G \geq 0$ and $C_{\varepsilon 3}=1$ if $G \leq 0$. In the ( $k$-w) SST model (Menter, 1994), the equation 6 is solved for $k$, and the dissipation $\omega$ is a ratio between $\varepsilon$ and $k$ so called specific turbulence dissipation $(\omega=\varepsilon / k)$.

\subsection{Governing equations of fluid-particle interaction}

The essential base of the combined of discrete approach (DEM) with continuum approach (CFD), is the interaction force between the fluid and the solid particles. This 
interaction force consists of two forces; buoyancy force and drag force. The buoyancy force is produced accuse the fluid pressure gradient around each individual particle (Kafui et al., 2011), expressed in this work as,

$F_{i}^{B}=\frac{4 \pi}{3} r_{i}^{3} \rho_{f} g$

While the drag force is the viscous shearing stresses effect of fluid on the particle. The drag force (Helland et al., 2000) is expressed by:

$F_{i}^{D}=\frac{\pi}{2} C_{D, i} r_{i}^{2} \rho_{f}\left(\vec{v}_{f}-\vec{v}_{i}\right)^{2} \varepsilon^{2-n}$

where $C_{D, i}$ is the drag coefficient, $\varepsilon_{i}^{-n}$ is porosity correction function to consider the effect of presence of other particles in neighbors of the particle $i$, and $n$ is exponent, it is an important factor in fluid-solid flows and educe from the experimental results on sedimentation and fluidization of Richardson \& Zaki, (1954) :

$\frac{v_{r}}{v_{o, i}}=\varepsilon_{i}^{n}$

where $v_{r}$ is the relative settling velocity between the fluid and the particle, $v_{o, i}$ is the terminal falling velocity of the particle and is obtained from Stokes' law of sedimentation (Stokes, 1901), and expressed as,

$v_{o, i}=\frac{g d_{i}^{2}\left(\rho_{i}-\rho_{f}\right)}{18 \mu_{f}}$

The drag force coefficient used herein the coupling DEM-CFD is the correlation proposed by Brown and Lawler, (2003), expressed as:

$C_{D, i}=\frac{24}{R e_{i}}\left(1+0.15 R e_{i}^{0.681}\right)+\frac{0.407}{1+\frac{8710}{R e_{i}}}$

where $\operatorname{Re}_{i}=\rho_{f} \varepsilon_{i} d_{i}\left|v_{f}-v_{i}\right| / \mu_{f}$ is the particle Reynolds number, $d_{i}$ is the diameter of the particle, and $\mu_{f}$ is the dynamic viscosity.

The interstitial velocity of the fluid is evaluated from the velocity calculation by CFD code:

$\vec{v}_{f}=K \frac{\vec{v}}{\varepsilon_{i}}$

where $K$ is a geometric factor that takes into account including the tortuosity of the solid phase (Gilbilaro, 2001). The tortuosity is the ratio of the actual path length through the pores to the euclidean distance, $\left(K=l_{\text {actual }} / l_{\text {euclidean }}\right)$. Other thing, the calculation of the 
porosity in this work is calculated by REV method (Representative Element Volume), which centered on the particle (Sherko, 2017), and expressed as, $\varepsilon=\frac{V_{R E V}-V_{i, R E V}}{V_{R E V}}$

where $V_{R E V}$ is the volume of the REV and calculated as, $\left(V_{R E V}=\left(l_{r} \times l_{r}\right) \times d_{i}, \quad l_{r}=y \times d_{i}, \quad y=1,2,3, . e t c.\right)$ and $V_{i, R E V}$ is the volumes of the particles in the REV $\left(V_{i, R E V}=N \times V_{i}\right)$, where $N$ is the number of particles in the REV, and $V_{i}$ is the volume of particle.

\section{Results}

\subsection{Validation of the coupling DEM-CFD}

A numerical simulation of one particle $\left(d_{i}=2 \mathrm{~mm}\right)$ in a parallelepiped, which is designed by using Salome platform (version 7), each side of the base has a length of 25 times the diameter of sphere $\left(d_{i}=2 \mathrm{~mm}\right)$, therefore the base is $(0.05 \mathrm{~m} \times 0.05 \mathrm{~m})$ to prevent the effect of the walls with fluid flow in z-direction, and a height of $1.0 \mathrm{~m}$, the geometry is shown in Fig.2. The physical parameters used in the simulation are listed in Table 1.

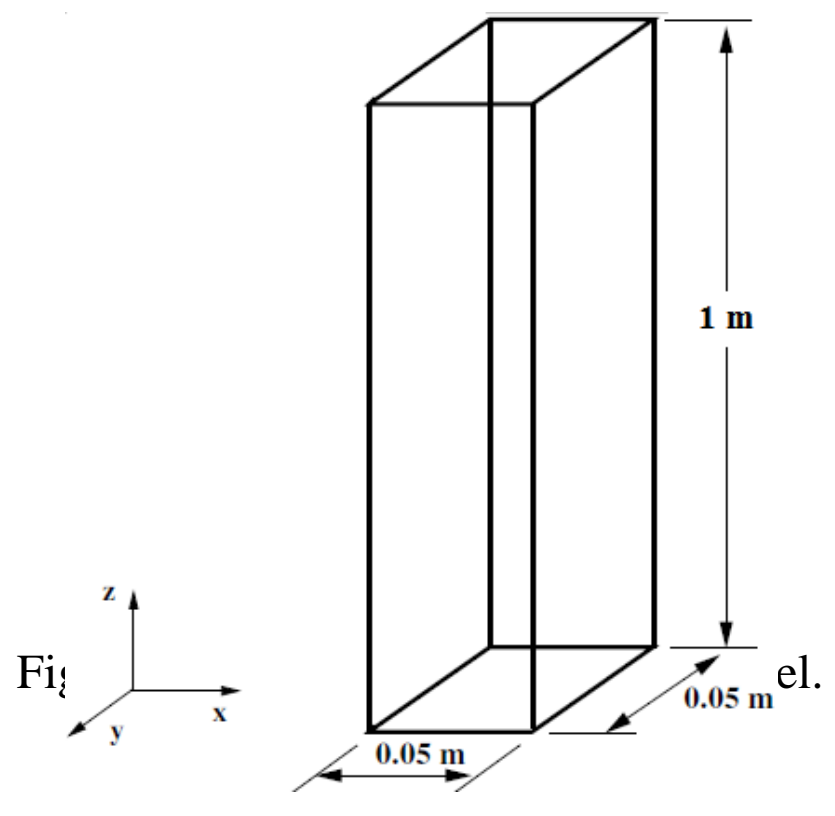


Table 1: Physical parameters used in the simulation.

\begin{tabular}{|l|c|}
\hline Parameter & Value \\
\hline Particle diameter $[\mathrm{mm}]$ & 2 \\
\hline Density of particle $\left[\mathrm{kg} / \mathrm{m}^{3}\right]$ & 2500 \\
\hline Young's modulus $[\mathrm{GPa}]$ & 70 \\
\hline Poisson coefficient & 0.35 \\
\hline Coefficient of friction & 0.3 \\
\hline Coefficient of restitution & 0.9 \\
\hline Density of water $\left[\mathrm{kg} / \mathrm{m}^{3}\right]$ & 1000 \\
\hline Dynamic viscosity of water $[\mathrm{Pa} . \mathrm{s}]$ & 0.001 \\
\hline DEM time step $[\mathrm{s}]$ & $1 \times 10^{-5}$ \\
\hline
\end{tabular}

\subsection{The relationship between the hydrodynamics characteristics}

To analyze the behaviors of fluid-solid particle interactions, it is necessary to understand the relationships between the hydrodynamics characteristics of the fluidized bed, such as the drag force, porosity of the bed, tortuosity, and height of the bed in the fluidization process. The relation between the tortuosity and the size of REV is depicted in Fig.3, it shows that value of the tortuosity is decreasing and approaches to 1 when the size of REV is increased.

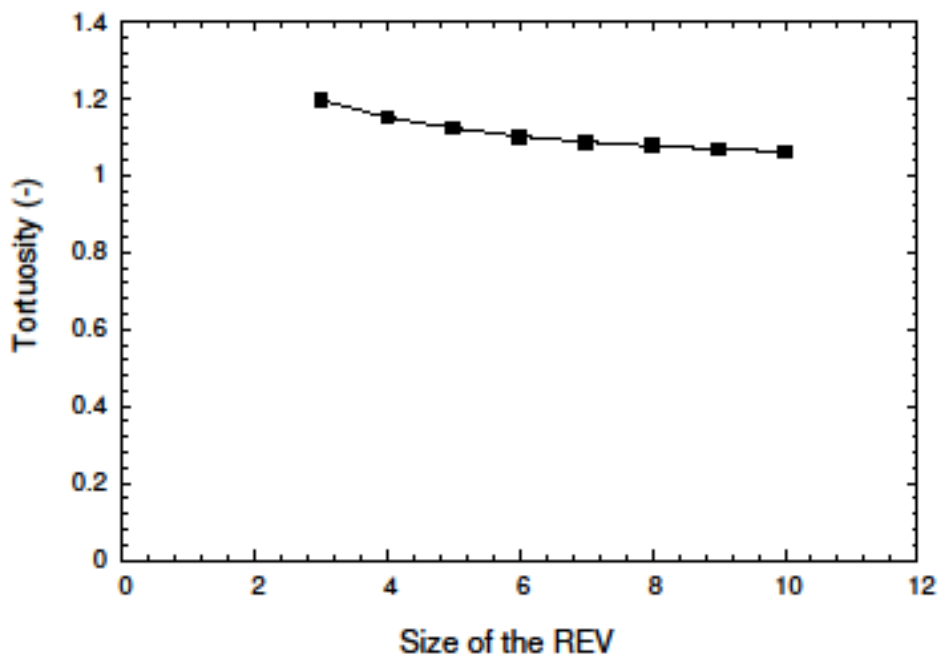

Figure 3: Relationship between the tortuosity and the size of REV.

In other hand, Fig. 4 depicted that the value of porosity for the fluid is increased with the increase the size of REV. From Fig.4, it is obvious that size of REV beyond the size of (5x5), changing the size of REV does not significantly change the value of porosity approaching to 1 . The numerical results show that the values of drag force increase with the increase of velocity of the inlet fluid. The results for a single sphere with $d_{i}=2 \mathrm{~mm}$, 
are presented in Fig. 5, a comparison between the results of theoretical drag force calculated from equation 15, with several models that used (laminar, $k-\varepsilon$ and $k-\omega$ SST).

$$
F_{t h}^{D}=\frac{1}{2} C_{D, i} \rho_{f} \pi r_{i}^{2} v_{r}^{2}
$$

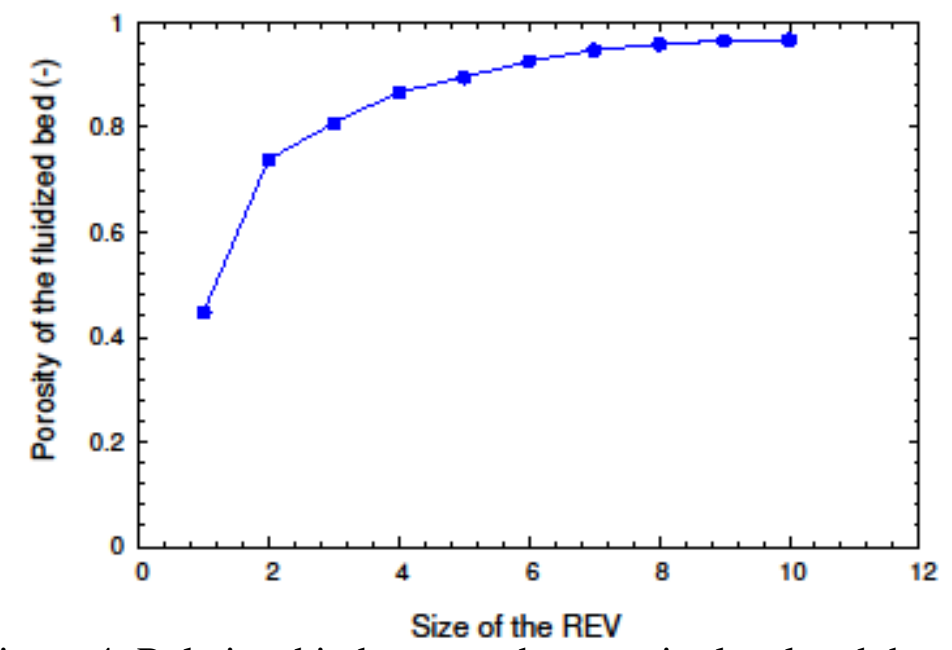

Figure 4: Relationship between the porosity local and the size of REV.

The curves in Fig.5, are depicted that the curve of drag force of $k-\varepsilon$ model is the nearest to the theoretical curve of drag force.

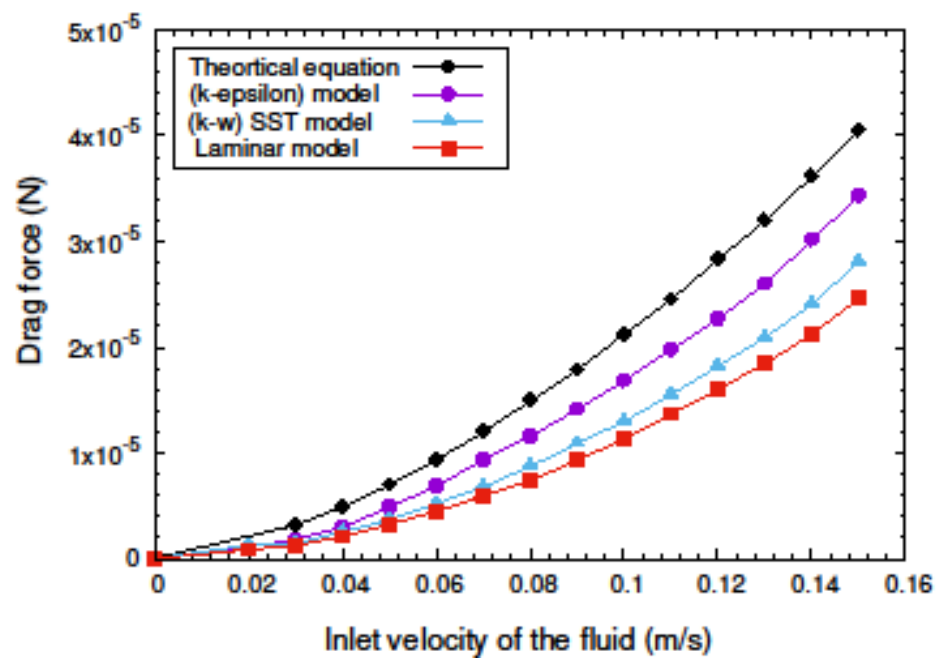

Figure 5: Relation between the drag force and inlet velocity of the fluid.

The Fig. 6 depicts the values of exponent of porosity, which are calculated from equation 10 , are plotted with different inlet velocity of the fluid for different sizes of REV. The four curves show that the value of the exponent doesn't depend on the inlet velocity but is strongly dependent on the sizes of REV. 


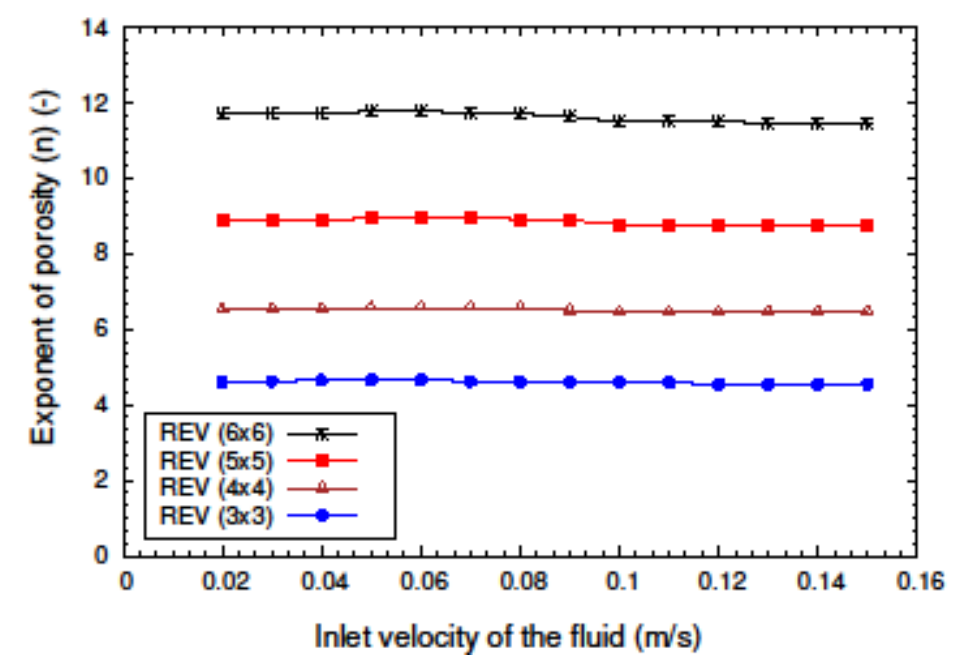

Figure 6: Relationship between the exponent of porosity and the inlet velocity of the fluid

\subsection{Results of coupling DEM-CFD (fluidization)}

In order to attest the feasibility of the DEM-CFD model, a comparison for the numerical simulation of coupling DEM-CFD model with experimental data (A1Arkawazi et al., 2017) has been done. For this purpose, a geometry of fluidizing column (pipe) with dimensions; diameter of the pipe $(\mathrm{D}=96 \mathrm{~mm})$ and height $(\mathrm{H}=1 \mathrm{~m})$, as depicted in (Fig.7). The physical parameters used in the simulation are listed in Table 2.

Then, two size of REV (3x3), with exponent of porosity $(n=4.6)$, and REV $(4 \times 4)$, with exponent of porosity $(n=6.5)$ are tested. In the fluid side, $k-\varepsilon$ model for a range of inlet water velocity $(0-0.14 \mathrm{~m} / \mathrm{s})$ has been used. The results are plotted in Fig. 7; the results of size of REV ( $3 \times 3)$ are the nearest and consistent with the experimental results more than the other size of REV $(4 \times 4)$.

Table 2: Physical parameters of the simulation.

\begin{tabular}{|l|c|}
\hline Parameter & Value \\
\hline Particle diameter [mm] & 2 \\
\hline Number of particles & 2640 \\
\hline Density of particle $\left[\mathrm{kg} / \mathrm{m}^{3}\right]$ & 2500 \\
\hline Young's modulus [GPa] & 70 \\
\hline Poisson coefficient & 0.35 \\
\hline \multicolumn{1}{|c|}{ Coefficient of friction } & 0.3 \\
\hline Coefficient of restitution & 0.9 \\
\hline Density of water $\left[\mathrm{kg} / \mathrm{m}^{3}\right]$ & 1000 \\
\hline Dynamic viscosity of water [Pa.s] & 0.001 \\
\hline DEM time step [s] & $1 \times 10^{-5}$ \\
\hline
\end{tabular}




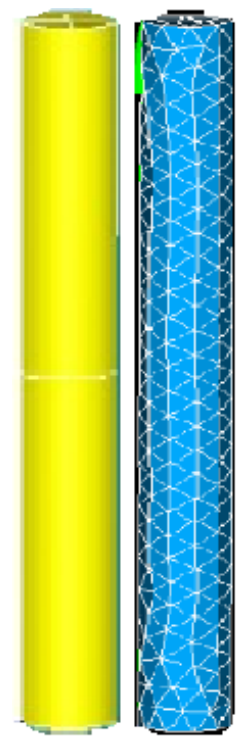

(a) (b)

Figure 7: Sketch of fluidized bed, a) geometry 3D, b) meshing

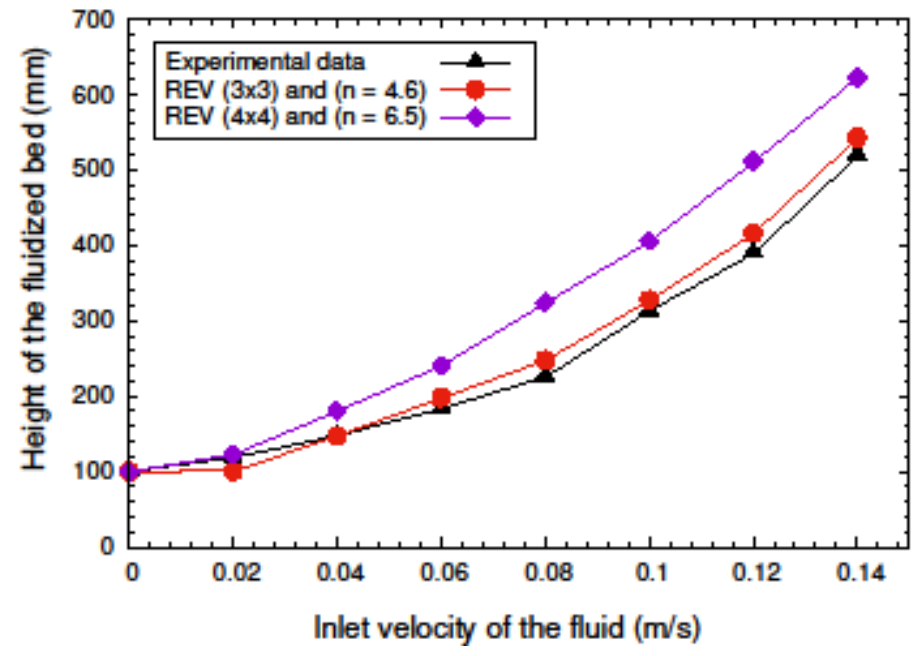


Figure 8: Comparison between the numerical results with the experimental results. Then, an example of simulation (coupling DEM-CFD) for the fluidization process has been done. The snapshots of fluidization process are presented in Fig. 9, for REV size (3x3) with exponent of porosity $(n=4.6)$, and inlet velocity of the water is $0.12 \mathrm{~m} / \mathrm{s}$. The bed is initially at static $(\mathrm{t}=0)$, then the flow of the water from the inlet of the pipe in upward direction causes fluidization of the particles, as it can be seen the bed start to expanded at $\mathrm{t}=0.25 \mathrm{~s}$, and the bed expansion is maximal at about $\mathrm{t}=5.0 \mathrm{~s}$ and the height of fluidized bed is remained fluctuating at this height as shown in the Fig. 9 for the rest of the time. It can notice in Fig. 9 that a heterogeneous distribution of particles in the cylinder, that can be related to a forming of clusters that mounting upward the fluidized bed before falling back slightly.

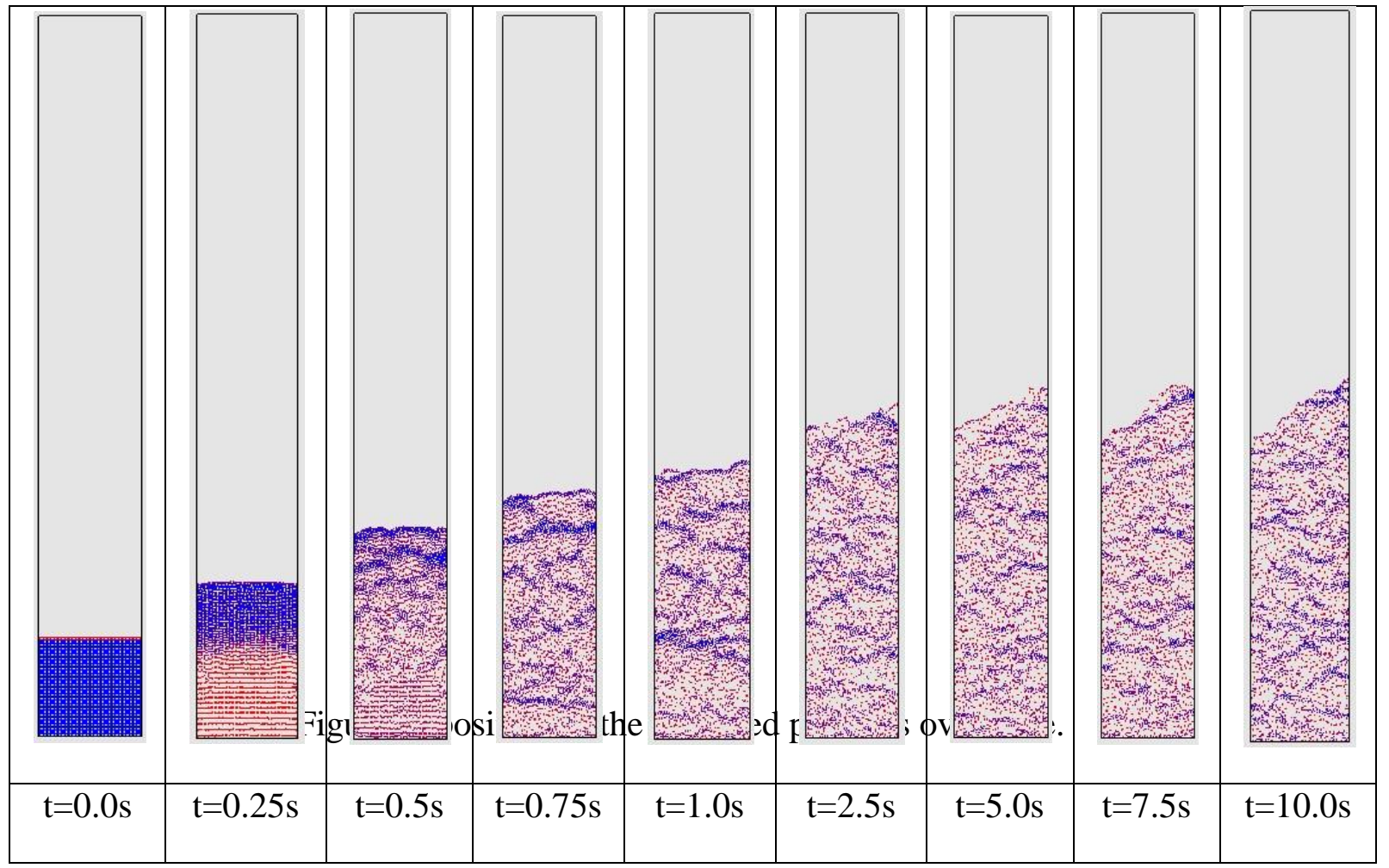

\section{Discussion}

The study presents an attempt in analyzing and modeling the hydrodynamic behavior of a fluidized bed by means of a coupling between a discrete element code (DEM), and Computational Fluid Dynamics (CFD) calculation. The action of the fluid on the particles is expressed in terms of drag force requiring a good estimation of the exponent of porosity, which is considering the group effects of particles on the global and local flow structures in a fluid-particle circulating fluidized bed. For this reason, we studied 
the influence of the size of the REV (Representative Volume Element), tortuosity and porosity on the value of the exponent of porosity. The comparison between the numerical results with the experimental ones, attests of a very good agreement. We found that the best size of REV is $(3 \times 3)$ with the exponent of porosity $(n=4.6)$, which approach to other values of $(n)$ that proposed by other researchers, for example Helland et al., (2007) who proposed $(n=4.7)$. The good results of the coupling DEM-CFD model for the fluidized bed, will allow us in the longer term; to add heat transfer models and mass transfer ones between the fluid and particles in simulations.

\section{References}

Matheson, G. L., Herbst, W. A., \& Halt, P. H. (1949). Characteristics of Fluid-Solid Systems. Industrial Engineering Chemical, 41, 1099.

Richardson, J.F., \& Zaki, W.N. (1954).Sedimentation and fluidization: part I. Trans. Inst. Chem. Eng. 32, 35-53.

Hartge, E., \& Werther, J. (1986 ). Analysis of the local structure of the two-phase flow in a fast fluidized bed. In, Circulating Fluidized Bed Technology. Chemical Engineering Technology, 58, 153-160.

Bürger, R., \&Wendland, W.L. (2001). Sedimentation and suspension flows: historical perspective and some recent developments. Journal Engineering Mathematic, 41 (2), 101-116.

Hirano, Y., Kai, T., Tsutsui, T., \& Nakazato, T. (2013). Decrease in the fluidization quality of fluidized beds containing binary mixtures of different catalyst particles. Chemical Engineering Science, 96 (7), 98-105.

Tsuji, Y., Kawaguchi, T., \& Tanaka, T. (1993). Discrete particle simulation of two dimensional fluidized beds. Powder Technology, 77, 79-87.

Hoomans, B.P.B., Kuipers, J.A.M., Briels, W.J., \& Van Swaaij, W.P.M. (1996). Discrete particle simulation of bubble and slug formation in a two-dimensional gasfluidized bed: a hard- sphere approach. Chemical Engineering Science, 51, 99-118.

Yu, A.B., \& Xu, B.H. (2003). Particle-scale modelling of gas-solid flow in fluidisation. J. Chem. Technol. Biotechnol, 78, 111-121.

Zhu, H.P., Zhou, Z.Y., Yang, R.Y., \& Yu, A.B. (2008). Discrete particle simulation of particulate systems: a review of major applications and findings. Chemical Engineering Science, 63, 5728-5770. 
Chavan, P. V., Thombare, M. A., Bankar, S. B., Kalaga, D. V., \& Patil-Shinde, V. A. (2018). Novel multistage solid-liquid circulating fluidized bed: Hydrodynamic characteristics. Particuology, 38, 134-142.

Di Maio, F. P., \& Di Renzo, A. (2007). DEM-CFD Simulations of fluidized beds with application in mixing dynamics. KONA Journal, 25, 205-216.

Gidaspow, D. (1994). Multiphase flow and fluidization, Academic Press, NewYork.

Apostolou, K., \& Hrymak, A.N. (2008). Discrete element simulation of liquid-particle flows. Computers and Chemical Engineering, 32, 841-856.

Wang, J.W., van der Hoef, M.A., \& Kuipers, J.A.M. (2010). CFD study of the minimum bubbling velocity of Geldart A particles in gas-fluidized beds. Chemical Engineering Science, 65, 3772-3785.

Chu, K.W., Kuang, S.B., Zhou, Z.Y., Yu A.B. (2018). Model A vs. Model B in the modelling of particle-fluid flow. Powder Technology, 329, 47-54.

Al-Arkawazia, S., Marieb, C., Benhabibb, K., \& Coorevits, P. (2017). Modeling the hydrodynamic forces between fluid-granular medium by coupling DEM-CFD. Chemical Engineering Research and Design, 117, 439-447.

Deen, N.G., Van Sint Annaland, M., Van der Hoef, M.A., \& Kuipers, J.A.M. (2007). Review of discrete particle modeling of fluidized beds. Chemical Engineering Science, 62, 28-44.

Fortin, J., \& De Saxcé, G. (1999). Modélisation numérique des milieux granulaires par l'approche du bi-potentiel. C. R. Academic Science, 327,721-724.

Archambeau, F., Méchitoua, N., \& Sakiz, M. (2004). Code Saturne: a finite volume method for the computation of turbulent incompressible flow-industrial applications. Int. J. Finite, 1 (1), 1-62.

Menter, F.R. (1994). Two-equation eddy-viscosity turbulence models for engineering applications. AIAA Journal, 32(8), 1598-1605.

Mohammadi, B., \& Pironneau, O. (1994). Analysis of the k- $\varepsilon$ turbulence model, J. Wiley \& Sons.

Kafui, D.K., Johnson, S., Thornton, C., \& Seville, J.P.K. (2011). Parallelization of a Lagrangian-Eulerian DEM/CFD code for application to fluidized beds. Powder Technology, 207 (1-3), 270-278.

Helland, E. , Occelli, R., \&Tadrist, L. (2000). Numerical study of cluster formation in a gas-particle circulating fluidized bed. Powder Technology, 110, 210-221.

KloStokes, G.G. (1901). Mathematical and Physical Papers. Cambridge University Press, Cambridge.

Brown, P., \& Lawler, D. (2003). Sphere drag and settling velocity revisited. 
Journal Environment Engineering, 129 (3), 222-231.

Gilbilaro, L.G. (2001). Fluidization-Dynamics: The Formulation and Applications of a Predictive Theory for the Fluidized State. Butterworth-Heinemann.

Sherko Ahmad Flamarz (2017). Comparison of porosity models for fluidized beds. Kurdistan Journal of Applied Research, 2 (1), 74-83.

Helland, E., Bournot, H., Occelli, R., \& Tadrist, L. (2007). Drag reduction and cluster formation in a circulating fluidized bed. Chemical Engineering Science, 62, 148-158. 